\title{
A mechanism of supernova explosion driven by magnetic monopoles
}

\author{
Qiu-He Peng ${ }^{1}$, Jing-Jing, Liu ${ }^{2}$ and Chih-Kang Chou ${ }^{3}$ \\ ${ }^{1}$ Department of Astronomy, Nanjing University, Nanjing, Jiangshu 210000, China \\ email: qhpeng@nju.edu.cn \\ ${ }^{2}$ College of Marine Science and Technology, Hainan Tropical Ocean University, Sanya, Hainan \\ 572022 , China \\ email: liujjingjing68@126.com \\ ${ }^{3}$ National Astronomical Observatory, Chinese Academy of Sciences, Beijing, 100000, China
}

\begin{abstract}
Taking Rubakov-Callen effect (that nucleons may decay catalyzed by magnetic monopoles) as the energy source, we propose a unified model of supernova driven by magnetic monopoles in this paper.
\end{abstract}

Keywords. supernovae, magnetic monopoles, nuclear reactions.

\section{Introduction}

Some predications of our model of huge massive star with magnetic monopoles (hereafter MMs) (Peng et al. (2001)) have been confirmed in recent years. Especially, our prediction of the radial magnetic field is also in good agreement with the lower limit of $8 \mathrm{mG}$ determined from the observations in 2013(Eatough et al. (2013)). Besides, our predication for the production of large amount of positrons from the galactic core is quantitatively verified by the observations in 2003 (Knodlseder (2003)). These observations are some new possible anticipated astronomical signals for existence of magnetic monopoles (Peng et al. (2016), Peng et al. (2017)).

\section{Overview}

As for possible applications to astrophysics, the most important property of MMs is that these exotic particles may induce nucleon decay (called Rubakov- Callen effect or RC effect), i.e., $p M \rightarrow e^{+} \pi^{0} M+$ debris (85\%), $\mathrm{pM} \rightarrow \mathrm{e}^{+} \mu^{ \pm} \mathrm{M}+$ debris (15\%). The MMs accumulated at the central core of the various types of the supernovae are originate from (MMs) captured in flight by their progenitors, so their number is directly proportional to both the surface area and the life time of the progenitors mainly during their red giant stage.

$$
N_{m}=4 \pi R^{2} \phi_{m} t \approx 1.0 \times 10^{31}\left(\frac{\phi_{m}^{0}}{10^{-2} \phi_{m}^{\mathrm{up}}}\right)\left(\frac{R_{\mathrm{RG}}}{10^{3} R_{\odot}}\right)^{2}\left(\frac{t_{\mathrm{RG}}}{10^{6} \mathrm{Yr}}\right),
$$

where $R_{\mathrm{RG}}$ denotes the radius of the star in its red giant stage and $t_{\mathrm{RG}}$ is the life time during the red giant stage of the progenitor of supernova. $\phi_{m}^{\text {up }} \approx 10^{-14} \mathrm{~cm}^{-2} \mathrm{~s}^{-1} \mathrm{sr}^{-1}$ is the upper limit of the flux of the MMs in space.

The total luminosity generated by the nucleon decay induced by MMs in the central region of various stellar objects may the estimated as

$$
L_{m} \approx \frac{4 \pi}{3} r_{c}^{3} n_{m} n_{\mathrm{B}}\left\langle\sigma \nu_{\mathrm{T}}\right\rangle m_{\mathrm{B}} c^{2}=N_{m} n_{B}\left\langle\sigma \nu_{\mathrm{T}}\right\rangle m_{\mathrm{B}} c^{2} .
$$


We now substitute the number of MMs accumulated at the central core of the various types of supernovae from Eq.(2.1) into Eq.(2.2) to obtain

$$
L_{m} \approx 2.5 \times 10^{43} a\left(\frac{\xi}{10^{2}}\right)\left(\frac{n_{\mathrm{B}}^{c}}{n_{\text {nuc }}}\right)\left(\frac{T_{c}}{10^{11} \mathrm{~K}}\right)^{1 / 2} \mathrm{ergs} / \mathrm{s},
$$

where $a=\left(R_{\mathrm{RG}} /\left(10^{3} R_{\odot}\right)\right)^{2}\left(t_{\mathrm{RG}} /\left(10^{6} \mathrm{Yr}\right), \xi \equiv \sigma /\left(10^{-30} \mathrm{~cm}^{3}\right)\left(\phi_{\mathrm{m}}^{0} /\left(10^{-2} \phi_{\mathrm{m}}^{\mathrm{up}}\right)\right)\right.$. Although the two factors, $\sigma /\left(10^{-30} \mathrm{~cm}^{3}\right),\left(\phi_{\mathrm{m}}^{0} /\left(10^{-2} \phi_{\mathrm{m}}^{\mathrm{up}}\right)\right)$ are uncertain, but the typical value of the parameter $\xi$ in Eq.(2.3) may be taken as $\xi \approx 100$ by comparing the heat flux for hot molten interior of the Earth with the same Eq.(2.3) in the unified model .

\section{Implications}

The masses for the progenitors SNII, SNIB, SNIC, SLSN are respectively given by $(8-25) M_{\odot},(30-60) M_{\odot},(80-150) M_{\odot}$, and $(200-1000) M_{\odot}$. In order to explode the entire star violently, the RC luminosity must much exceed the Eddington's luminosity. That is $L \gg L_{E d d}=(4 \pi c G M) / \kappa \approx 1.3 \times 10^{38}(\kappa / 0.4)^{-1}\left(M / M_{\odot}\right)$ ergs/s, where $\kappa$ denotes opacity. When $L \gg L_{E d d}$ is satisfied, the corresponding radiation pressure is so huge that the stellar mantle, outer layer and the stellar atmosphere of the entire star are all violently ejected outwards. The highest speed of the drastic projection may reach $(1-2) \times 10^{4} \mathrm{~km} / \mathrm{s}$. This is the observed scenario of supernovae explosion. As for how large the ratio of $L_{m} / L_{\mathrm{Edd}}$ must be in order to achieve supernovae explosion, detailed numerical simulation is really needed. If the RC luminosity is not much higher than the Eddington's luminosity of the star during the collapse of the central core, then the resulting explosion is rather weak and may be referred to as dark explosion. For an examples, the corresponding supernovae of the supernovae remnant Cas $\mathrm{A}$ and the interstellar nebula G1.9 + 0.3 which is observed by the Chandra X-ray satellite and is considered as a supernova remnant exploded about 110 years age. More recently, NASA reported that a star N6946-BH1 suddenly disappears from astronomical observations in 2015 (http : //inews.ifeng.com/51176539/news.shtml?srctag $=$ pc2m\&back). It is an typical example for the dark explosion. An important result is that the remnant of supernovae explosion is a neutron star rather than a black hole even though the mass of the supernovae being huge due to still there being a few magnetic monopoles in the central region of the remnant and they continue catalyze the nucleons decay.

\section{Acknowledgements}

This work was supported in part by the National Natural Science Foundation of China under grants 11565020, 10773005, and the Counterpart Foundation of Sanya under grant 2016PT43, the Special Foundation of Science and Technology Cooperation for Advanced Academy and Regional of Sanya under grant 2016YD28, the Scientific Research Starting Foundation for 515 Talented Project of Hainan Tropical Ocean University under grant RHDRC201701, and the Natural Science Foundation of Hainan Province under grant 114012 .

\section{References}

Eatough, R., P. 2013, Nature, 591, 391

Kn ödlseder, J., et al. 2003, A\&A, 411, 457

Peng Q. H. \& Chou C. K. 2001, ApJ, 551, 23

Peng Q. H., Liu J. J., \& Chou C. K 2016, Ap\&SS, 361, 388,

Peng Q. H., Liu J. J., \& Ma Z. Q. 2017, ApJ, 57, 59 